This postprint is published in:

Mediterranean journal of mathematics, Volume 17, Issue 4, August 2020, art. 111, 9

DOI: 10.1007/s00009-020-01551-1

\title{
LOW PERTURBATIONS FOR A CLASS OF NONUNIFORMLY ELLIPTIC PROBLEMS
}

\author{
ANOUAR BAHROUNI AND DUŠAN D. REPOVŠ
}

\begin{abstract}
We introduce and study a new functional which was motivated by our paper on the Caffarelli-Kohn-Nirenberg inequality with variable exponent (Bahrouni, Rădulescu \& Repovš, Nonlinearity 31 (2018), 1518-1534). We also study the eigenvalue problem for equations involving this new functional.
\end{abstract}

\section{Introduction}

The Caffarelli-Kohn-Nirenberg inequality plays an important role in studying various problems of mathematical physics, spectral theory, analysis of linear and nonlinear PDEs, harmonic analysis, and stochastic analysis. We refer to Chaudhuri \& Ramaswamy [2], Baroni, Colombo \& Mingione [4], Colasuonno \& Pucci [7], and Colombo \& Mingione [8] for relevant applications of the CaffarelliKohn-Nirenberg inequality.

Let $\Omega \subset \mathbb{R}^{N}(N \geq 2)$ be a bounded domain with smooth boundary. The following CaffarelliKohn-Nirenberg inequality (see Caffarelli, Kohn \& Nirenberg [5]) establishes that given $p \in(1, N)$ and real numbers $a, b$, and $q$ such that

$$
-\infty<a<\frac{N-p}{p}, \quad a \leq b \leq a+1, \quad q=\frac{N p}{N-p(1+a-b)},
$$

there is a positive constant $C_{a, b}$ such that for every $u \in C_{c}^{1}(\Omega)$,

$$
\left(\int_{\Omega}|x|^{-b q}|u|^{q} d x\right)^{p / q} \leq C_{a, b} \int_{\Omega}|x|^{-a p}|\nabla u|^{p} d x .
$$

This inequality has been extensively studied (see, e.g. Abdellaoui \& Peral [1], Chaudhuri \& Ramaswamy [2], Bahrouni, Rădulescu \& Repovš [3], Catrina \& Wang [6], and Mihăilescu, Rădulescu $\&$ Stancu [11], and the references therein).

In particular, Bahrouni, Rădulescu \& Repovš [3] gives a new version of the Caffarelli-KohnNirenberg inequality with variable exponent. The next theorem is proved under the following assumptions: let $\Omega \subset \mathbb{R}^{N}(N \geq 2)$ be a bounded domain with smooth boundary and suppose that the following hypotheses are satisfied

(A) $a: \bar{\Omega} \rightarrow \mathbb{R}$ is a function of class $C^{1}$ and there exist $x_{0} \in \Omega, r>0$, and $s \in(1,+\infty)$ such that:

(1) $|a(x)| \neq 0$, for every $x \in \bar{\Omega} \backslash\left\{x_{0}\right\}$;

(2) $|a(x)| \geq\left|x-x_{0}\right|^{s}$, for every $x \in B\left(x_{0}, r\right)$;

(P) $p: \bar{\Omega} \rightarrow \mathbb{R}$ is a function of class $C^{1}$ and $2<p(x)<N$ for every $x \in \Omega$.

Theorem 1.1. (see Bahrouni, Rădulescu \& Repovš [3]) Suppose that hypotheses $(A)$ and $(P)$ are satisfied. Let $\Omega \subset \mathbb{R}^{N}(N \geq 2)$ be a bounded domain with smooth boundary. Then there exists a

Key words and phrases. Caffarelli-Kohn-Nirenberg inequality, eigenvalue problem, critical point theorem, generalized Lebesgue-Sobolev space, Luxemburg norm.

2010 Math. Subj. Classif.: Primary 35J60, Secondary 35J91, 58E30. 
positive constant $\beta$ such that

$$
\begin{aligned}
\int_{\Omega}|a(x)|^{p(x)}|u(x)|^{p(x)} d x & \leq\left.\beta \int_{\Omega}|a(x)|^{p(x)-1}|| \nabla a(x)|| u(x)\right|^{p(x)} d x \\
& +\beta\left(\int_{\Omega}|a(x)|^{p(x)}|\nabla u(x)|^{p(x)} d x+\int_{\Omega}|a(x)|^{p(x)}|\nabla p(x)||u(x)|^{p(x)+1} d x\right) \\
& +\beta \int_{\Omega}|a(x)|^{p(x)-1}|\nabla p(x)||u(x)|^{p(x)-1} d x .
\end{aligned}
$$

for every $u \in C_{c}^{1}(\Omega)$.

Motivated by Bahrouni, Rădulescu \& Repovš [3], we introduce and study in the present paper a new functional $T: E_{1} \rightarrow \mathbb{R}$ via the Caffarelli-Kohn-Nirenberg inequality, in the framework of variable exponents. More precisely, we study the eigenvalue problem in which functional $T$ is present. Our main result is Theorem 4.2 and we prove it in Section 5.

\section{Function spaces with variable exponent}

We recall some necessary properties of variable exponent spaces. We refer to Hajek, Santalucia, Vanderwerff \& Zizler [10], Musielak [12], Papageorgiou, Rădulescu \& Repovš [13], Rădulescu [15], Rădulescu [16], and Rădulescu \& Repovš [17], and the references therein.

Consider the set

$$
C_{+}(\bar{\Omega})=\{p \in C(\bar{\Omega}) \mid p(x)>1 \text { for all } x \in \bar{\Omega}\} .
$$

For any $p \in C_{+}(\bar{\Omega})$, let

$$
p^{+}=\sup _{x \in \bar{\Omega}} p(x) \quad \text { and } \quad p^{-}=\inf _{x \in \bar{\Omega}} p(x),
$$

and define the variable exponent Lebesgue space as follows

$$
L^{p(x)}(\Omega)=\left\{u \mid u \text { is measurable real-valued function such that } \int_{\Omega}|u(x)|^{p(x)} d x<\infty\right\},
$$

with the Luxemburg norm

$$
|u|_{p(x)}=\inf \left\{\mu>\left.0\left|\int_{\Omega}\right| \frac{u(x)}{\mu}\right|^{p(x)} d x \leq 1\right\} .
$$

We recall that the variable exponent Lebesgue spaces are separable and reflexive Banach spaces if and only if $1<p^{-} \leq p^{+}<\infty$, and continuous functions with compact support are dense in $L^{p(x)}(\Omega)$ if $p^{+}<\infty$.

Let $L^{q(x)}(\Omega)$ denote the conjugate space of $L^{p(x)}(\Omega)$, where $1 / p(x)+1 / q(x)=1$. If $u \in L^{p(x)}(\Omega)$ and $v \in L^{q(x)}(\Omega)$ then the following Hölder-type inequality holds:

$$
\left|\int_{\Omega} u v d x\right| \leq\left(\frac{1}{p^{-}}+\frac{1}{q^{-}}\right)|u|_{p(x)}|v|_{q(x)} .
$$

An important role in manipulating the generalized Lebesgue-Sobolev spaces is played by the $p($.$) - modular of the L^{p(x)}(\Omega)$ space, which is the mapping $\rho: L^{p(x)}(\Omega) \rightarrow \mathbb{R}$ defined by

$$
\rho(u)=\int_{\Omega}|u|^{p(x)} d x .
$$

Proposition 2.1. (see Rădulescu \& Repovš [17]) The following properties hold

(i) $|u|_{p(x)}<1($ resp.,$=1 ;>1) \Leftrightarrow \rho(u)<1($ resp.,$=1 ;>1)$;

(ii) $|u|_{p(x)}>1 \Rightarrow|u|_{p(x)}^{p^{-}} \leq \rho(u) \leq|u|_{p(x)}^{p^{+}}$; and

(iii) $|u|_{p(x)}<1 \Rightarrow|u|_{p(x)}^{p^{+}} \leq \rho(u) \leq|u|_{p(x)}^{p^{-}}$. 
Proposition 2.2. (see Rădulescu \& Repovš [17]) If $u, u_{n} \in L^{p(x)}(\Omega)$ and $n \in \mathbb{N}$, then the following statements are equivalent

(1) $\lim _{n \rightarrow+\infty}\left|u_{n}-u\right|_{p(x)}=0$.

(2) $\lim _{n \rightarrow+\infty} \rho\left(u_{n}-u\right)=0$.

(3) $u_{n} \rightarrow u$ in measure in $\Omega$ and $\lim _{n \rightarrow+\infty} \rho\left(u_{n}\right)=\rho(u)$.

We define the variable exponent Sobolev space by

$$
W^{1, p(x)}(\Omega)=\left\{u \in L^{p(x)}(\Omega)|| \nabla u \mid \in L^{p(x)}(\Omega)\right\} .
$$

On $W^{1, p(x)}(\Omega)$ we consider the following norm

$$
\|u\|_{p(x)}=|u|_{p(x)}+|\nabla u|_{p(x)} .
$$

Then $W^{1, p(x)}(\Omega)$ is a reflexive separable Banach space.

\section{Functional $T$}

We shall introduce a new functional $T: E_{1} \rightarrow \mathbb{R}$ motivated by the Caffarelli-Kohn-Nirenberg inequality obtained in Bahrouni, Rădulescu \& Repovš [3].

We denote by $E_{1}$ the closure of $C_{c}^{1}(\Omega)$ under the norm

$$
\begin{aligned}
\|u\|= & \|\left.\left. B(x)\right|^{\frac{1}{p(x)}} \nabla u(x)\right|_{p(x)}+\left|A(x)^{\frac{1}{p(x)}} u(x)\right|_{p(x)}+ \\
& \|\left.\left. D(x)\right|^{\frac{1}{p(x)+1}} u(x)\right|_{p(x)+1}+\left.\left.|| C(x)\right|^{\frac{1}{p(x)-1}} u(x)\right|_{p(x)-1},
\end{aligned}
$$

where the potentials $A, B, C$, and $D$ are defined by

$$
\left\{\begin{array}{l}
A(x)=|a(x)|^{p(x)-1}|\nabla a(x)| \\
B(x)=|a(x)|^{p(x)} \\
C(x)=|a(x)|^{p(x)-1}|\nabla p(x)| \\
D(x)=B(x)|\nabla p(x)|
\end{array}\right.
$$

We now define $T: E_{1} \rightarrow \mathbb{R}$ as follows

$$
\begin{aligned}
T(u) & =\int_{\Omega} \frac{B(x)}{p(x)}|\nabla u(x)|^{p(x)} d x+\int_{\Omega} \frac{A(x)}{p(x)}|u(x)|^{p(x)} d x \\
& +\int_{\Omega} \frac{D(x)}{p(x)+1}|u(x)|^{p(x)+1} d x+\int_{\Omega} \frac{C(x)}{p(x)-1}|u(x)|^{p(x)-1} d x .
\end{aligned}
$$

The following properties of $T$ will be useful in the sequel.

Lemma 3.1. Suppose that hypotheses $(A)$ and $(P)$ are satisfied. Then the functional $T$ is welldefined on $E_{1}$. Moreover, $T \in C^{1}\left(E_{1}, \mathbb{R}\right)$ with the derivative given by

$$
\begin{aligned}
\langle L(u), v\rangle=\left\langle T^{\prime}(u), v\right\rangle & =\int_{\Omega} B(x)|\nabla u(x)|^{p(x)-2} \nabla u(x) \nabla v(x) d x+\int_{\Omega} A(x)|u(x)|^{p(x)-2} u(x) v(x) d x \\
& +\int_{\Omega} D(x)|u(x)|^{p(x)-1} u(x) v(x) d x+\int_{\Omega} C(x)|u(x)|^{p(x)-3} u(x) v(x) d x,
\end{aligned}
$$

for every $u, v \in E_{1}$.

Proof. The proof is standard, see Rădulescu \& Repovš [17].

Lemma 3.2. Suppose that hypotheses $(A)$ and $(P)$ are satisfied. Then the following properties hold (i) $L: E_{1} \rightarrow E_{1}^{*}$ is a continuous, bounded and strictly monotone operator;

(ii) $L$ is a mapping of type $\left(S_{+}\right)$, i.e. if $u_{n} \rightarrow u$ in $E_{1}$ and

$$
\limsup _{n \rightarrow+\infty}\left\langle L\left(u_{n}\right)-L(u), u_{n}-u\right\rangle \leq 0,
$$

then $u_{n} \rightarrow u$ in $E_{1}$. 
Proof. (i) Evidently, $L$ is a bounded operator. Recall the following Simon inequalities (see Simon $[18])$ :

$$
\begin{cases}|x-y|^{p} \leq c_{p}\left(|x|^{p-2} x-|y|^{p-2} y\right) \cdot(x-y) & \text { for } p \geq 2 \\ |x-y|^{p} \leq C_{p}\left[\left(|x|^{p-2} x-|y|^{p-2} y\right) \cdot(x-y)\right]^{\frac{p}{2}}\left(|x|^{p}+|y|^{p}\right)^{\frac{2-p}{2}} & \text { for } 1<p<2\end{cases}
$$

for every $x, y \in \mathbb{R}^{N}$, where

$$
c_{p}=\left(\frac{1}{2}\right)^{-p} \text { and } C_{p}=\frac{1}{p-1} .
$$

Using inequalities (4) and recalling that $2<p^{-}$, we can prove that $L$ is a strictly monotone operator.

(ii) The proof is identical to the proof of Theorem 3.1 in Fan \& Zhang [9].

\section{Main theorem}

We recall our Compactness Lemma:

Lemma 4.1. (see Bahrouni, Rădulescu \& Repovš [3]) Suppose that hypotheses $(A)$ and $(P)$ are satisfied and that $p^{-}>1+s$. Then $E_{1}$ is compactly embeddable in $L^{q}(\Omega)$ for each $q \in\left(1, \frac{N p^{-}}{N+s p^{+}}\right)$. Moreover, the same conclusion holds if we replace $L^{q}(\Omega)$ by $L^{q(x)}(\Omega)$, provided that $q^{+}<\frac{N p^{-}}{N+s p^{+}}$.

We are concerned with the following nonhomogeneous problem

$$
\left\{\begin{array}{l}
-\operatorname{div}\left(B(x)|\nabla u|^{p(x)-2} \nabla u\right)+\left(A(x)|u|^{p(x)-2}+C(x)|u|^{p(x)-3}\right) u= \\
\left(\lambda|u|^{q(x)-2}-D(x)|u|^{p(x)-1}\right) u \text { in } \Omega, \\
u=0 \text { on } \partial \Omega,
\end{array}\right.
$$

where $\lambda>0$ is a real number and $q$ is continuous on $\bar{\Omega}$. We assume that $q$ satisfies the following basic inequalities

$$
1<\min _{x \in \bar{\Omega}} q(x)<\min _{x \in \bar{\Omega}}(p(x)-1)<\max _{x \in \bar{\Omega}} q(x)<\frac{N p^{-}}{N+s p^{+}} .
$$

We can now state the main result of this paper.

Theorem 4.2. Suppose that all hypotheses of Lemma 4.1 are satisfied and that inequalities $(Q)$ hold. Then there exists $\lambda_{0}>0$ such that every $\lambda \in\left(0, \lambda_{0}\right)$ is an eigenvalue for problem (5).

In order to prove Theorem 4.2 (this will be done in the Section 5 ), we shall need some preliminary results. We begin by defining the functional $I_{\lambda}: E_{1} \rightarrow \mathbb{R}$,

$$
\begin{aligned}
I_{\lambda}(u) & =\int_{\Omega} \frac{B(x)}{p(x)}|\nabla u(x)|^{p(x)} d x+\int_{\Omega} \frac{A(x)}{p(x)}|u(x)|^{p(x)} d x+\int_{\Omega} \frac{C(x)}{p(x)-1}|u(x)|^{p(x)-1} d x \\
& +\int_{\Omega} \frac{D(x)}{p(x)+1}|u(x)|^{p(x)+1} d x-\lambda \int_{\Omega} \frac{|u(x)|^{q(x)}}{q(x)} d x .
\end{aligned}
$$

Standard argument shows that $I_{\lambda} \in C^{1}\left(E_{1}, \mathbb{R}\right)$ and

$$
\begin{aligned}
\left\langle I_{\lambda}^{\prime}(u), v\right\rangle & =\int_{\Omega} B(x)|\nabla u(x)|^{p(x)-2} \nabla u(x) \nabla v(x) d x+\int_{\Omega} A(x)|u(x)|^{p(x)-2} u(x) v(x) d x \\
& +\int_{\Omega} D(x)|u(x)|^{p(x)-1} u(x) v(x) d x+\int_{\Omega} C(x)|u(x)|^{p(x)-3} u(x) v(x) d x \\
& -\lambda \int_{\Omega}|u(x)|^{q(x)-2} u(x) v(x),
\end{aligned}
$$

for every $u, v \in E_{1}$.

Thus the weak solutions of problem (5) coincide with the critical points of $I_{\lambda}$. 
Lemma 4.3. Suppose that all hypotheses of Theorem 4.2 are satisfied. Then there exists $\lambda_{0}>0$ such that for any $\lambda \in\left(0, \lambda_{0}\right)$ there exist $\rho, \alpha>0$ such that

$$
I_{\lambda}(u) \geq \alpha \text { for any } u \in E_{1} \text { with }\|u\|=\rho .
$$

Proof. By Lemma 4.1, there exists $\beta>0$ such that

$$
|u|_{r(x)} \leq \beta\|u\|, \text { for every } u \in E_{1} \text { and } r^{+} \in\left(1, \frac{N p^{-}}{N+s p^{+}}\right) .
$$

We fix $\rho \in\left(0, \min \left(1, \frac{1}{\beta}\right)\right)$. Invoking Proposition 2.1, for every $u \in E_{1}$ with $\|u\|=\rho$, we can get

$$
|u|_{q(x)}<1 .
$$

Combining the above relations and Proposition 2.1, for any $u \in E_{1}$ with $\|u\|=\rho$, we can then deduce that

$$
\begin{aligned}
I_{\lambda}(u) & \geq \frac{1}{p^{+}}\left(\int_{\Omega} B(x)|\nabla u(x)|^{p(x)} d x+\int_{\Omega} A(x)|u(x)|^{p(x)} d x\right) \\
& +\frac{1}{p^{+}+1} \int_{\Omega} D(x)|u(x)|^{p(x)+1} d x \\
& +\frac{1}{p^{+}-1} \int_{\Omega} C(x)|u(x)|^{p(x)-1} d x-\frac{\lambda}{q^{-}} \int_{\Omega}|u(x)|^{q(x)} d x \\
& \geq \frac{1}{4^{p^{+}}\left(p^{+}+1\right)}\|u\|^{p^{+}+1}-\lambda \frac{\beta^{q^{-}}}{q^{-}}\|u\|^{q^{-}} \\
& \geq \frac{1}{4^{p^{+}}\left(p^{+}+1\right)} \rho^{p^{+}+1}-\lambda \frac{\beta^{q^{-}}}{q^{-}} \rho^{q^{-}} \\
& =\rho^{q^{-}}\left(\frac{1}{4^{p^{+}\left(p^{+}+1\right)}} \rho^{p^{+}+1-q^{-}}-\lambda \frac{\beta^{q^{-}}}{q^{-}}\right) .
\end{aligned}
$$

Put $\lambda_{0}=\frac{\rho^{p^{+}+1-q^{-}}}{4^{p^{+}}\left(2 p^{+}+2\right)} \frac{q^{-}}{\beta^{q^{-}}}$. It now follows from (6) that for any $\lambda \in\left(0, \lambda_{0}\right)$,

$$
I_{\lambda}(u) \geq \alpha \text { with }\|u\|=\rho,
$$

and $\alpha=\frac{\rho^{p^{+}+1}}{4^{p^{+}}\left(2 p^{+}+2\right)}>0$. This completes the proof of Lemma 4.3.

Lemma 4.4. Suppose that all hypotheses of Theorem 4.2 are satisfied. Then there exists $\varphi \in E_{1}$ such that $\varphi>0$ and $I_{\lambda}(t \varphi)<0$, for small enough $t$.

Proof. By virtue of hypotheses $(P)$ and $(Q)$, there exist $\epsilon_{0}>0$ and $\Omega_{0} \subset \Omega$ such that

$$
q(x)<q^{-}+\epsilon_{0}<p^{-}-1, \text { for every } x \in \Omega_{0} .
$$

Let $\varphi \in C_{0}^{\infty}(\Omega)$ such that $\overline{\Omega_{0}} \subset \operatorname{supp}(\varphi), \varphi=1$ for every $x \in \overline{\Omega_{0}}$ and $0 \leq \varphi \leq 1$ in $\Omega$. It then follows that for $t \in(0,1)$,

$$
\begin{aligned}
I_{\lambda}(t \varphi) & =\int_{\Omega} \frac{t^{p(x)} B(x)}{p(x)}|\nabla \varphi(x)|^{p(x)} d x+\int_{\Omega} \frac{t^{p(x)} A(x)}{p(x)}|\varphi(x)|^{p(x)} d x+\int_{\Omega} \frac{t^{p(x)-1} C(x)}{p(x)-1}|\varphi|^{p(x)-1} d x \\
& +\int_{\Omega} \frac{t^{p(x)+1} D(x)}{p(x)+1}|\varphi(x)|^{p(x)+1} d x-\lambda \int_{\Omega} t^{q(x)} \frac{|\varphi(x)|^{q(x)}}{q(x)} d x \\
& \leq \frac{t^{p^{-}-1}}{p^{-}-1}\left(\int_{\Omega} \frac{B(x)}{p(x)}|\nabla \varphi(x)|^{p(x)} d x+\int_{\Omega} \frac{A(x)}{p(x)}|\varphi(x)|^{p(x)} d x+\int_{\Omega} \frac{C(x)}{p(x)-1}|\varphi|^{p(x)-1} d x\right. \\
& \left.+\int_{\Omega} \frac{D(x)}{p(x)+1}|\varphi(x)|^{p(x)+1} d x\right)-\lambda t^{q^{-}+\epsilon_{0}} \int_{\Omega} \frac{|\varphi(x)|^{q(x)}}{q(x)} d x .
\end{aligned}
$$

Combining (7) and (8), we finally arrive at the desired conclusion.

This completes the proof of Lemma 4.4. 


\section{Proof of Theorem 4.2}

In the last section we shall prove the main theorem of this paper.

Let $\lambda_{0}$ be defined as in Lemma 4.3 and choose any $\lambda \in\left(0, \lambda_{0}\right)$.

Again, invoking Lemma 4.3, we can deduce that

$$
\inf _{u \in \partial B(0, \rho)} I_{\lambda}(u)>0 .
$$

On the other hand, by Lemma 4.4, there exists $\varphi \in E_{1}$ such that

$$
I_{\lambda}(t \varphi)<0 \text { for every small enough } \mathrm{t}>0 .
$$

Moreover, by Proposition 2.1, when $\|u\|<\rho$, we have

$$
I_{\lambda}(u) \geq \frac{1}{4^{p^{+}}\left(p^{+}+1\right)}\|u\|^{p^{+}+1}-c\|u\|^{q^{-}},
$$

where $c$ is a positive constant. It follows that

$$
-\infty<m=\inf _{u \in B(0, \rho)} I_{\lambda}(u)<0 .
$$

Applying Ekeland's variational principle to the functional

$$
I_{\lambda}: B(0, \rho) \rightarrow \mathbb{R},
$$

we can find a (PS) sequence $\left(u_{n}\right) \in B(0, \rho)$, that is,

$$
I_{\lambda}\left(u_{n}\right) \rightarrow m \text { and } I_{\lambda}^{\prime}\left(u_{n}\right) \rightarrow 0 .
$$

It is clear that $\left(u_{n}\right)$ is bounded in $E_{1}$. Thus there exists $u \in E_{1}$ such that, up to a subsequence,

$$
\left(u_{n}\right) \rightarrow u \text { in } \mathrm{E}_{1} \text {. }
$$

Using Theorem 4.1, we see that $\left(u_{n}\right)$ strongly converges to $u$ in $L^{q(x)}(\Omega)$.

So, by the Hölder inequality and Proposition 2.2, we can obtain the following

$$
\lim _{n \rightarrow+\infty} \int_{\Omega}\left|u_{n}\right|^{q(x)-2} u_{n}\left(u_{n}-u\right) d x=\lim _{n \rightarrow+\infty} \int_{\Omega}|u|^{q(x)-2} u\left(u_{n}-u\right) d x=0 .
$$

On the other hand, since $\left(u_{n}\right)$ is a $(\mathrm{PS})$ sequence, we can also infer that

$$
\lim _{n \rightarrow+\infty}\left\langle I_{\lambda}^{\prime}\left(u_{n}\right)-I_{\lambda}^{\prime}(u), u_{n}-u\right\rangle=0 .
$$

Combining the above pieces of information with Lemma 3.2, we can now conclude that

$$
u_{n} \rightarrow u \text { in } \mathrm{E}_{1} .
$$

Therefore

$$
I_{\lambda}(u)=m<0 \text { and } I_{\lambda}^{\prime}(u)=0 .
$$

We have thus shown that $u$ is a nontrivial weak solution for problem (5) and that every $\lambda \in\left(0, \lambda_{0}\right)$ is an eigenvalue of problem (5).

This completes the proof of Theorem 4.2.

\section{Acknowledgements}

The second author was supported by the Slovenian Research Agency grants P1-0292, J1-7025, J18131, N1-0064, N1-0083, and N1-0114. We thank the referee for comments and suggestions. 


\section{References}

[1] B. Abdellaoui and I. Peral, Some results for quasilinear elliptic equations related to some Caffarelli-KohnNirenberg inequalities, Commun. Pure Appl. Anal. 2 (2003), 539-566.

[2] A.N. Chaudhuri, and M. Ramaswamy, An improved Hardy-Sobolev inequality and its application, Proc. Amer. Math. Soc. 130 (2002), 89-505.

[3] A. Bahrouni, V. Rădulescu and D.D. Repovš, A weighted anisotropic variant of the Caffarelli-Kohn-Nirenberg inequality and applications, Nonlinearity. 31 (2018), no. 4, 1516-1534.

[4] P. Baroni, M. Colombo, and G. Mingione, Non-autonomous functionals, borderline cases and related function classes, St. Petersburg Math. J. 27 (2016), 347-379.

[5] L. Caffarelli, R. Kohn, and L. Nirenberg, First order interpolation inequalities with weights, Compos. Math. 53 (1984), 259-275

[6] F. Catrina and Z.Q. Wang, On the Caffarelli-Kohn-Nirenberg inequalities: sharp constants, existence (and nonexistence) and symmetry of extremal function, Commun. Pure Appl. Math. 54 (2001), 229-258.

[7] F. Colasuonno and P. Pucci, Multiplicity of solutions for $p(x)$-polyharmonic elliptic Kirchhoff equations, Nonlinear Anal. 74 (2011), no. 17, 5962-5974.

[8] M. Colombo and G. Mingione, Bounded minimisers of double phase variational integrals, Arch. Ration. Mech. Anal. 218 (2015), 219-273.

[9] X. Fan and Q. Zhang, Existence of solutions for $p(x)$-Laplacian Dirichlet probem, Nonlinear Anal. 52 (2003), 1843-1852.

[10] P. Hajek, V.M. Santalucia, J. Vanderwerff, and V. Zizler, Biorthogonal Systems in Banach Spaces, CMC Books in Mathematics, Springer, New York, 2008

[11] M. Mihăilescu, V. Rădulescu, and D. Stancu, A Caffarelli-Kohn-Nirenberg type inequality with variable exponent and applications to PDE's, Complex Variables Elliptic Eqns. 56 (2011), 659-669.

[12] J. Musielak, Orlicz Spaces and Modular Spaces, Lecture Notes in Mathematics, Vol. 1034, Springer, Berlin, 1983.

[13] N.S. Papageorgiou, V.D. Rădulescu and D.D. Repovš, Nonlinear Analysis - Theory and Methods, Springer Monographs in Mathematics, Springer, Cham, 2019.

[14] P.H. Rabinowitz, Minimax Theorems and Applications to Nonlinear Partial Differential Equations, in: L.Cesari, R. Kannan, H.F. Weinberger (Eds.), Nonlinear Analysis, Academic Press, New York, 1978, pp. 161-177.

[15] V.D. Rădulescu, Nonlinear elliptic equations with variable exponent: old and new, Nonlinear Anal. 121 (2015), 336-369.

[16] V.D. Rădulescu, Isotropic and anisotropic double-phase problems: old and new, Opuscula Math. 39 (2019), 259-279.

[17] V.D. Rădulescu and D.D. Repovš, Partial Differential Equations with Variable Exponents: Variational Methods and Qualitative Analysis, CRC Press, Taylor \& Francis Group, Boca Raton, FL, 2015.

[18] J. Simon, Régularité de la solution d'une équation non linéaire dans $\mathbb{R}^{N}$, Journées d'Analyse Non Linéaire (Proc. Conf., Besançon, 1977), Lecture Notes in Math., 665, Springer, Berlin, 1978, pp. 205-227.

(A. Bahrouni) Mathematics Department, University of Monastir, Faculty of Sciences, 5019 Monastir, TUNISIA

Email address: bahrounianouar@yahoo.fr

(D.D. Repovš) Faculty of Education and Faculty of Mathematics and Physics, University of Ljubljana, \& Institute of Mathematics, Physics and Mechanics, 1000 Ljubljana, Slovenia

Email address: dusan.repovs@guest.arnes.si 